\title{
A 3D Computed Tomography Based Tool for Orthopedic Surgery Planning
}

\author{
João Ribeiro, Victor Alves, Sara Silva and Jaime Campos
}

\begin{abstract}
The preparation of a plan is essential for a surgery to take place in the best way possible and also for shortening patient's recovery times. In the orthopedic case, planning has an accentuated significance due to the close relation between the degree of success of the surgery and the patient recovering time. It is important that surgeons are provided with tools that help them in the planning task, in order to make it more reliable and less time consuming. In this paper, we present a 3D Computed Tomography based solution and its implementation as an OsiriX plugin for orthopedic surgery planning. With the developed plugin, the surgeon is able to manipulate a three-dimensional isosurface rendered from the selected imaging study (a CT scan). It is possible to add digital representations of physical implants (surgical templates), in order to evaluate the feasibility of a plan. These templates are STL files generated from CAD models. There is also the feature to extract new isosurfaces of different voxel values and slice the final 3D model according to a predefined plane, enabling a 2D analysis of the planned solution. Finally, we discuss how the proposed application assists the surgeon in the planning process in an alternative way, where it is possible to three-dimensionally analyze the impact of a surgical intervention on the patient.
\end{abstract}

João Ribeiro

CCTC - Computer Science and Technology Center, University of Minho, Braga, Portugal, e-mail: joaopedroribeiro@me.com

Victor Alves

CCTC - Computer Science and Technology Center, University of Minho, Braga, Portugal, e-mail: valves@di.uminho.pt

Sara Silva

CCTC - Computer Science and Technology Center, University of Minho, Braga, Portugal, e-mail: sara.isa.silva@gmail.com

Jaime Campos

CCTC - Computer Science and Technology Center, University of Minho, Braga, Portugal, e-mail: jaimefbc@gmail.com 


\section{Introduction}

The surgery's success is intimately related with its planning. The pre-operative planning consists in an evaluation supported by the clinical information and the patient's studies to establish a surgical procedure suitable to it. During the planning process, a group of steps are defined that increases the chances of a successful surgery, improving the communication between the surgeon and the other members of the surgery, e. g., nurses, and anesthetist [21].

One of the difficulties that a surgeon faces is the need of visually precept the impact that his planned surgery will have on the patient. In the case of orthopedic surgery, this is even more important, since the analysis of implant's placement (when necessary) is only possible in real time (surgery). Moreover, it is important to annotate the location / actual position of a pre-surgical implant, thereby reducing the risk inherent to any surgical intervention. Allowing the patient situation to be previously analyzed in detail and with greater time interval, the task of the orthopedic surgeon is facilitated, because the pressure of the decision making during surgery is reduced. A surgeon tends to become less formal when planning only based on his previous professional experience. However, there is a need to become more effective and rigorous in the planning of more complex surgeries [21, 20, 10]. Surgeons are continuously searching to improve their performance, increasing their accuracy levels.

One of the current trends are the three dimension reconstructions which are used in several sectors of activity, namely in healthcare, particularly in diagnostics [19, 5]. Currently, there are several companies that offer computer-assisted orthopedic surgery (CAOS) solutions to help surgeons plan the surgical intervention. However, some of them are still based on orthogonal X-ray views, which remove the three dimensionality of the tissues (e.g. organs, bones). Although others use Computed Tomography (CT) scans, it is not possible for the surgeon to add templates that represent the implants that will be used in surgery, not allowing a global view of the planned solution. Some of these tools are Orthoview, TraumaCad, SurgiCase, HipOp [16].

The Orthoview solution can be integrated with DICOM Picture Archiving and Communication Systems (PACS) and uses two orthogonal X-ray images. After importing these images, the user is able to add vector lines (always in 2D) representing real physical implants [4]. Nonetheless, the surgeon is unable to visualize the resultant model in a 3D space. Due to the impossibility to convert a 3D structure into $2 \mathrm{D}$ without losing details, it is impossible to analyze the fracture (if there is one) with detail [3]. After these steps the surgeon can generate a report to use at the surgery.

Another application called TraumaCad is available from VoyantHealth. This application is fairly similar to Orthoview, although it uses CT scans instead of X-rays. However, both applications use 2D implants models (templates). In the surgeon's perspective, these models are represented through vector lines which decreases the perception of the trauma. In TraumaCad, the 3D visualization only allows the analysis of the study in other angles. The surgeon can add surgical templates to the CT scan and position them by using a multi view approach for guidance. For this to be 
accomplished, the software reslices the CT scan by using a multi-planar algorithm, therefore enabling the user to analyze the same image in four different angles (Axial, Coronal, Sagittal and Oblique). Although not being a real 3D model representation, it is a major step when compared with the previously mentioned application [18].

SurgiCase is available from the Belgian company Materialise. It allows the preoperative planning with the help of an engineer, where the surgeon can create a 3D model of the planned resolution but this is achieved with a remote assistant's help. This assistant is an engineer from Materialise that will work with him in a cooperative way, in order to develop a plan for the surgery. So, the surgeon has little autonomy since he cannot add templates or test other procedures. Consequently, if he wants to change anything in the plan, he must contact the assistant [1].

There is a free software application for pre-operative planning developed by Istituti Ortopedici Rizzoli and CINECA, named HipOp. This application was conceived for the total hip replacement. The system imports a CT scan which defines a 3D anatomical space. The anatomical objects are represented through multiple views. The implants can be loaded by the surgeon and they are represented by their $3 \mathrm{D}$ model in the same space. However, it is not possible to move both components at the same time independently [8].

Steen and Widegren [17] in association with Sectra Medical Systems AB in Sweden presented a prototype to analyze the fit of implants. The application shades the implants depending on the distance between them and the bone. Aiming for the total hip replacement, it offers the possibility to measure its critical distances. There are some limitations towards its 3D environment. The 3D implant model and the $3 \mathrm{D}$ volume reconstruction of the $\mathrm{CT}$ study are rendered independently which causes failures in the transparency. Beyond that, it is not possible to intersect both components, in this case the implant model is always totally in front or behind the 3D CT volume. Thus, it is difficult to predict surgery outcome for the patient.

Some other planning solutions use professional image editing tools, such as Adobe Photoshop. By using tools typically design for digital image processing, the surgeons create an image of the final result [15]. Some publications point out that the success of the surgery increases using virtual reality techniques where surgeons can practice their surgeries. This kind of technology is normally associated with the training carried out by pilots and astronauts [10].

The main reason for the lack of software that merge a CT scan and templates representing the implants to be used in surgery is due to the difficulty of dealing with two different major graphic types representations: 3D bitmaps (voxels) and vector images. On one hand, the CT scan, which is a series of volume based images with same thickness and equally spaced (i.e. matrix of voxels). On the other hand, we have a template provided by an orthopedic implants manufacturer, which is vector type. This template is a virtual representation of a physical support and its structure is a set of arranged triangles. Due to these structurally different image types, the development of a solution that aggregates these two types together on a same plane is somewhat challenging. In order to visualize and analyze all the angles of the fracture, a surgeon needs to freely manipulate the templates on the patient's imaging studies. This can only be satisfactorily achieved with a 3D model. Since a 
CT scan is a series of images with the same thickness and equally spaced, it allows us to create the corresponding 3D model. This model, allows a greater viewing and understanding of the fracture extent (in the case of bone tissue) $[5,19]$.

The three main rendering techniques that enable the creation of a CT scan 3D model are Multi-planar Rendering (MPR), Volume Rendering (VR) and Surface Rendering (SR). The MPR is usually used when only weak computational resources are available because the processing required is lower. It is widely used whenever the goal is to visualize the imaging study through different planes simultaneously (e.g. Axial, Coronal, Sagittal and Oblique). The VR technique is used when the purpose is to visualize the entire volume. Images are created by projecting rays in the volume from a viewpoint (Ray Casting method) [14]. For each ray that intersects the volume (one or more voxels), color and opacity values are calculated and then represented as a pixel. This technique requires a huge amount of runtime calculations, which implies more powerful machines. SR is the technique that was used in this work. It is, by definition, the visualization of a 3D object from a set of isosurfaces. They are only made by points with the same intensity which in this case refers to the attenuation value for radiation using the Hounsfield's scale. It is widely used whenever the goal is to visualize structures close to each other (e.g. visualize the skull on a brain CT scan) [22]. These isosurfaces can be constructed by contours that are extracted from each slice in order to create a surface based on the volume's contour or by voxels where the isosurfaces are generated directly from voxels with a predefined value from the Hounsfield's scale. One of the algorithms used in this reconstruction is the Marching Cubes (MC) [2,9].

The surgeon using any of these techniques can extract more information about the study because he is given the capability to analyze it in all possible angles. Yet, he is unable to add templates to elaborate a plan for the surgery. In this study, we present a solution for the problem of structural differences between images. With the proposed solution, the surgeon can import a CT scan, generate a 3D surface from it and add 3D surgical templates on top of it. This means that 3D vector graphics can be merged with a 3D matrix (generated) surface. The present article is structured as follows: first, 3D Modeling principles are introduced, then it is presented the features and the operation of the proposed solution, finishing with conclusions and the future steps to improve the application.

\section{3D Modeling and Visualization}

Each CT scan produces a volume of data that can be manipulated. In order to extract the isosurfaces from the CT scan, the MC algorithm was chosen [9]. These isosurfaces are constituted by a polygonal mesh which was computed from a scalar field, i.e., set of voxels. The process starts with a given predefined Hounsfield Unit (HU) value from the original imaging study. The voxels that meet this threshold requirement are then used by the MC algorithm to construct the isosurface by marching iteratively with an imaginary cube through the $3 \mathrm{D}$ grid where the voxels are projected. 
Constructing the 3D model entails proceeding with the scalar field and evaluating each vertices of the cube in order to use the best polygons to represent the original surface. These vertices are then aggregated to form the final isosurface of a polygon mesh. A lookup table (Figure 1) is used by the MC algorithm in order to decide how to fuse the vertices and resolve ambiguity when choosing the points which belong to the polygon mesh surface. Since the first algorithm implementation, this table has been refined over the years to provide better results.
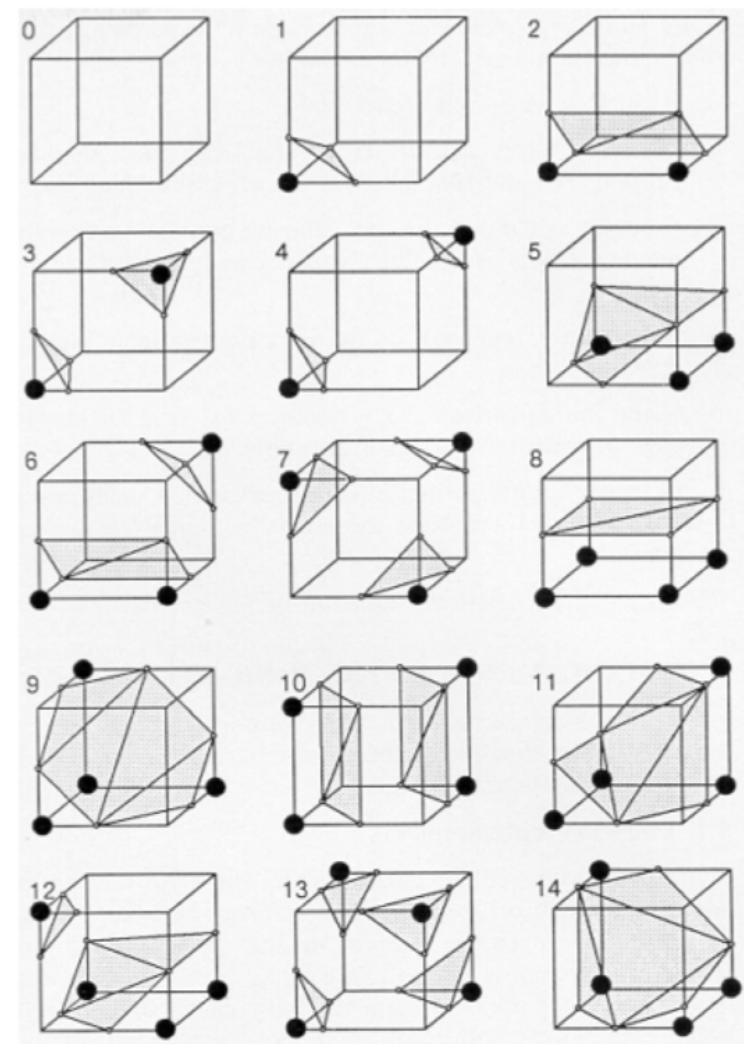

Fig. 1 The original published lookup table. 
Algorithm 1 presents the MC algorithm written in pseudocode.

Data: t-a predefined threshold in HU

Result: Set of triangles of the same value

for each image voxel do

a cube of length one is placed on eight adjacent voxels of the image;

for each of the cube's edge do

if the one of the node voxels has value greater than or equal to tand

the other voxel has value less than $t$ then

calculate the position of a point on the cube's edge that belongs to

the isosurface, using linear interpolation;

end

end

for each of the predefined cube configurations do

for each of the eight possible rotations do

for the configuration's complement do

compare the produced cube configuration of the above

calculated isopoints to the set of predefined cube

configurations and produce the corresponding triangles;

end

end

end

end

Algorithm 1: MC algorithm pseudocode

Each image (CT scan's slice) has associated metadata arranged by tags provided by the DICOM standard. This metadata contains information about the file, the patient it belongs to as well as the study. Among this information, some tags characterize the whole volume, like space between slices (tag 0018,0088), slice thickness (tag $0018,0050)$, slice location (tag 0020,1041) and number of slices (tag 0054,0081). Voxels parameters, such as position and thickness are set using the information provided by these tags. The MC algorithm then uses the voxels information to create the 3D models.

Figure 2 shows an example of a CT scan's slice, where its volumetric matrix structure is illustrated. The 3D representations of the surgical template's models are also polygon meshes, modeled after the original. Merging these models in the same graphic's scene along with the generated isosurfaces, will enable the visual intersection of both and give the ability to rotate and position each model independently. 


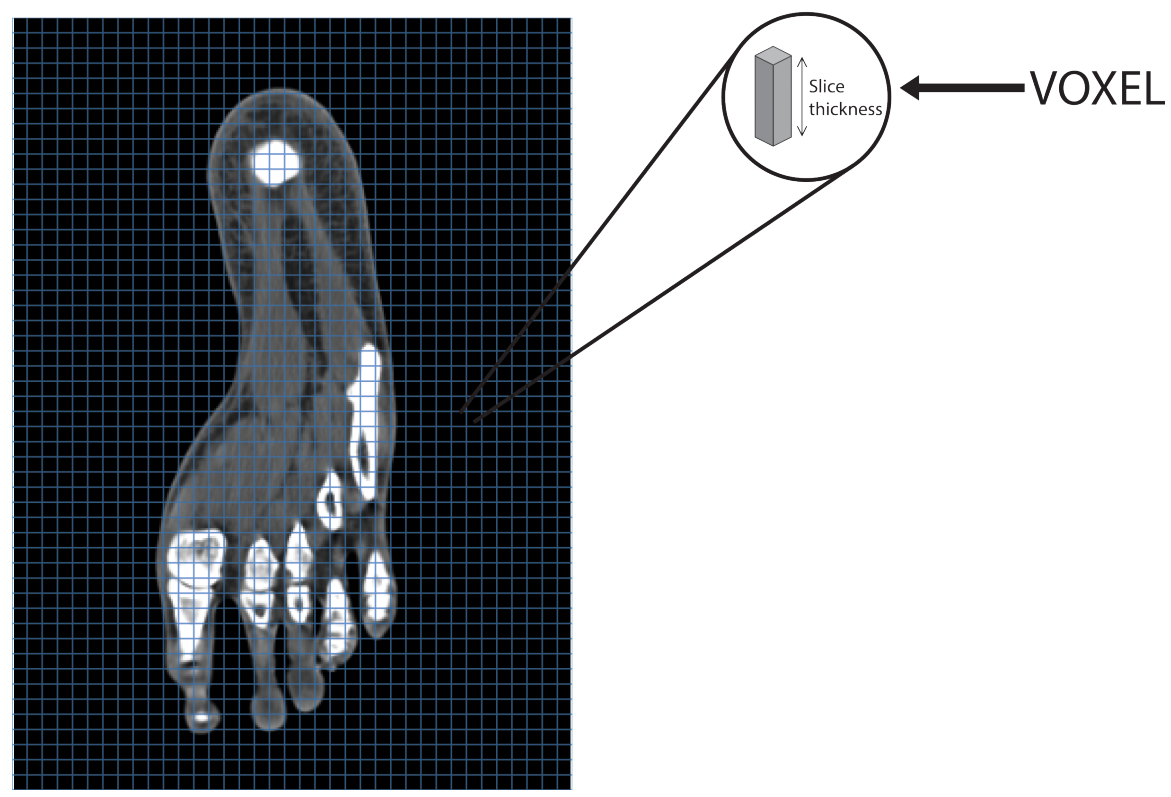

Fig. 2 Example of a CT scan's slice.

Since the surgeon is more familiar with the 2D representation of each slice, the application provides both ways of presenting the data. A MPR technique is used to help the surgeon better visualize the axial, coronal and sagittal planes of each CT scan's slice (Figure 3). When rendering in conjunction with the 3D generated model in the same scene, the surgeon is able to pan each 2D plane and visualize each slice representation independently in another viewer.
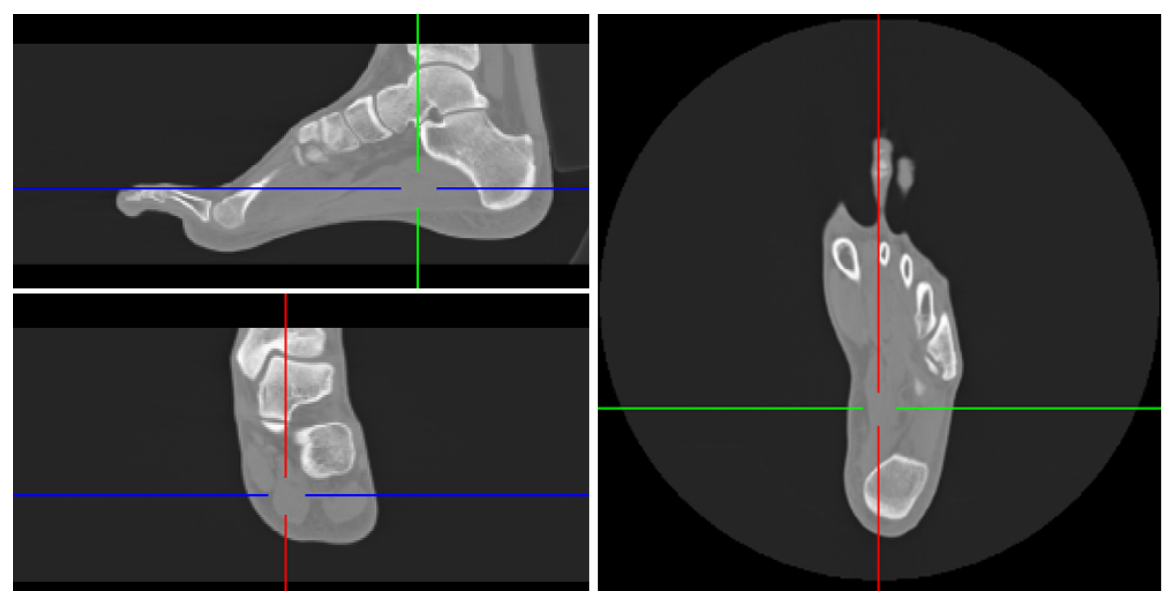

Fig. 3 OrthoMED Multi-planar Rendering visualization. 


\section{The OrthoMED plugin}

The proposed solution, which we named OrthoMED, was developed in $\mathrm{C}++$ and Objective-C as a plugin for OsiriX $[12,13]$ using a set of open source libraries written in the same programming language. They are OsiriX DCM Framework, used to read the DICOM files and their metadata, ITK and GDCM, to parse and process each DICOM file and VTK, to implement the MC algorithm and 3D visualization $[11,6,7]$. OsiriX was chosen since it is a widely used viewer for medical purposes, allowing a minimized learning curve associated with the usage of this new tool.

Figure 4 presents the application's internal workflow to create an isosurface from a CT scan. For OsiriX to detect OrthoMED as a plugin it is necessary to create a sub-class of Pluginfilter. That way it is possible to open the plugin from the OsiriX's main menu and import the CT scan from its ViewerController. Then, to read and extract meta-information from de CT scan (e.g., Patient's name, Patient's age) the OsiriX DCM Framework was used. By creating an instance from DCMObject all the meta-information associated to the CT study can be retrieved. With ITK and the GDCM libraries, the CT scan can be read and converted to a VTK object to be displayed on screen.

VTK has an implementation of the MC algorithm that when executed results in an isosurface of chosen HU where the templates can later be added.

Figure 5 presents the application's workflow, beginning with the CT scan's upload and ending with the report export. Since the isosurfaces and the templates are now structurally compatible, with VTK they can be resized and its position changed in the same geometric plane.

The proposed solution workflow can be divided in six steps:

Step A Select the imaging study (i.e. CT scan) and run the OrthoMED plugin. This step is done within the OsiriX environment;

Step B The current imaging study is transferred to the plugin that as a first interaction asks for the initial HU value for the isosurface to be created. Once this value is entered, the isosurface is generated and displayed. This isosurface is extracted using the MC algorithm;

Step C Add surgical templates and handle them, changing their positions in a 3D space. These templates model the implants that will be used in the surgery;

Step D Add different isosurfaces, enabling the analysis of existing intersections in the surrounding tissues;

Step E Slice the generated model that includes all the surgical templates. These slices are made in a chosen plane and in the initial isosurface;

Step F Export the report with the patient's info, a list of the physical implants used, selected images and notes that the surgeon included.

During Step C the surgeon is able to visualize the patient's CT scan with MPR, allowing its analysis in three different planes. By moving the related plane, the surgeon is now able to visualize each slice from the chosen plan. This is very helpful because it allows the precise location of the fracture. 


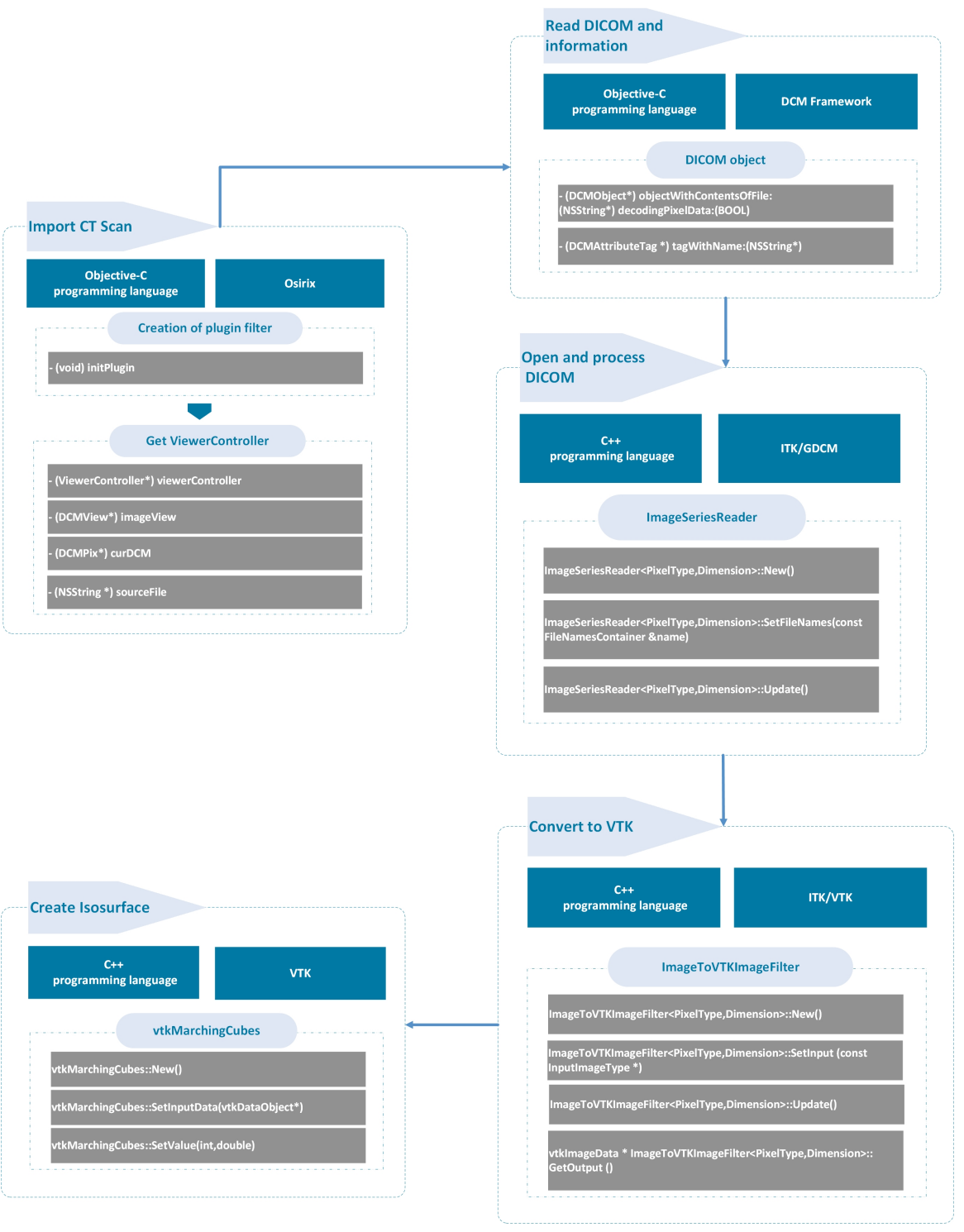

Fig. 4 OrthoMED's internal workflow to create an isosurface from a CT scan. 


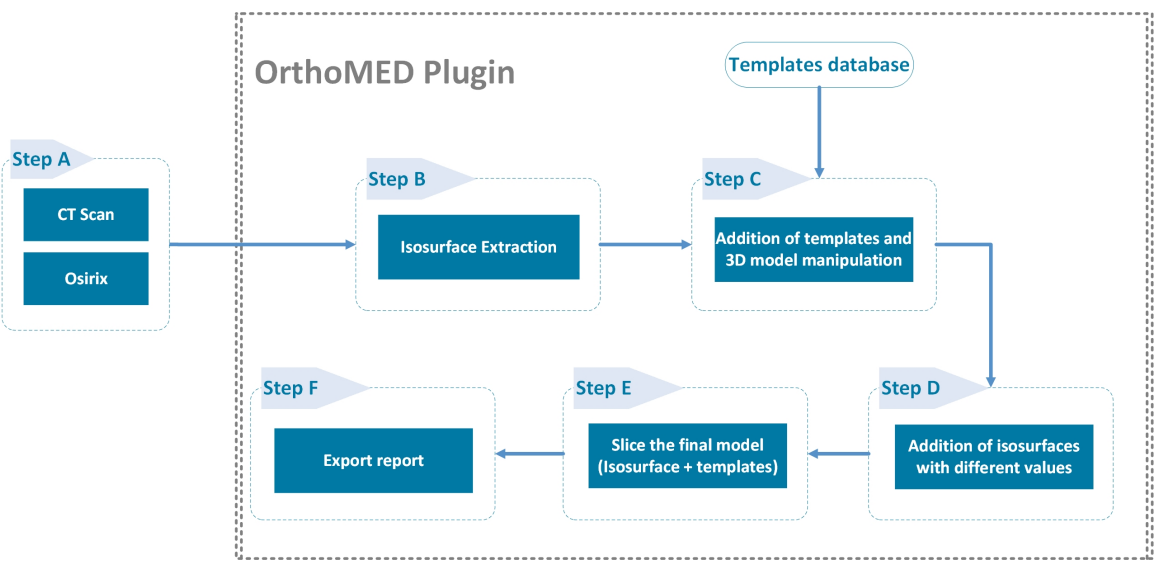

Fig. 5 OrthomeD's workflow.

Figure 6 presents a screenshot of OrthoMED's main window with its five sections:

a) Opens the window with the surgical templates database, where the surgeon can choose which template he wants to add. These templates are the 3D digital representation of a real template. They have the same shape and size;

b) Table with the surgical templates added, with their corresponding positions and angles;

c) Section where the final 3D model slices are displayed. Scrolling up and down shows the whole array;

d) Main section, where the surgeon handles the 3D isosurface as well as the templates, always in a 3D space;

e) Exporting section of the report with the surgery plan. 


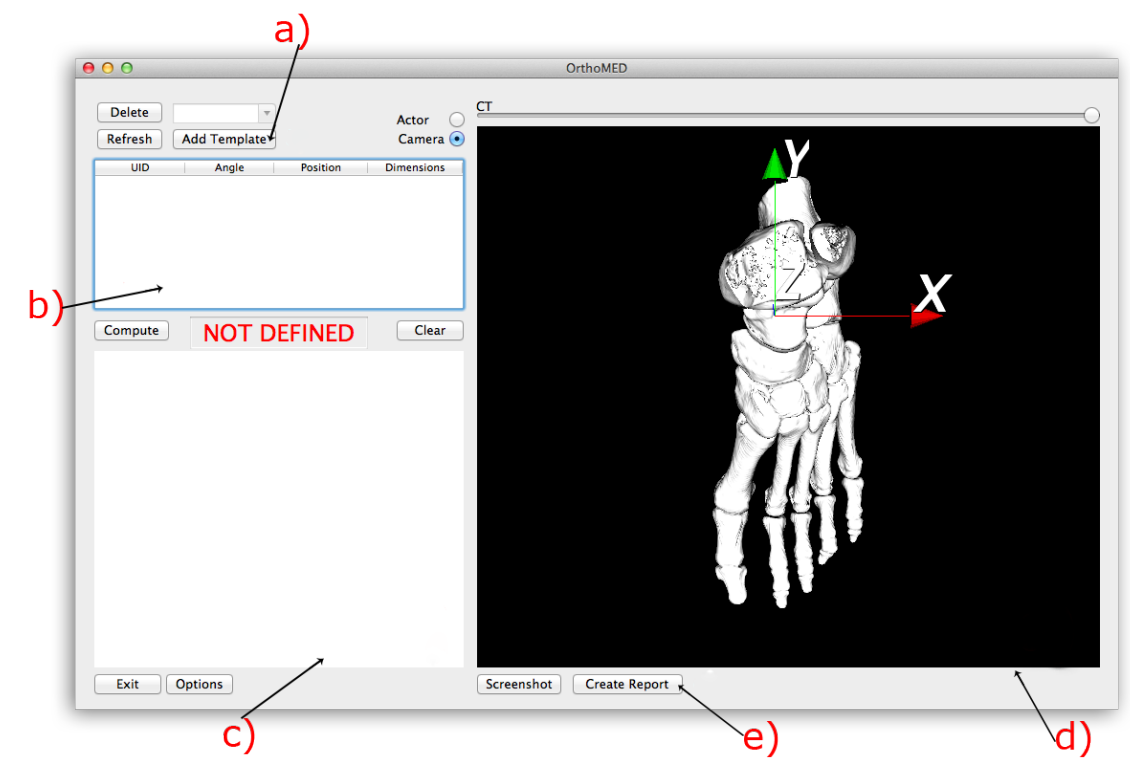

Fig. 6 OrthoMED's main window.

The upper slider can be used to change the isosurface's opacity, helping the visualization of the internal interceptions (Figure 7).

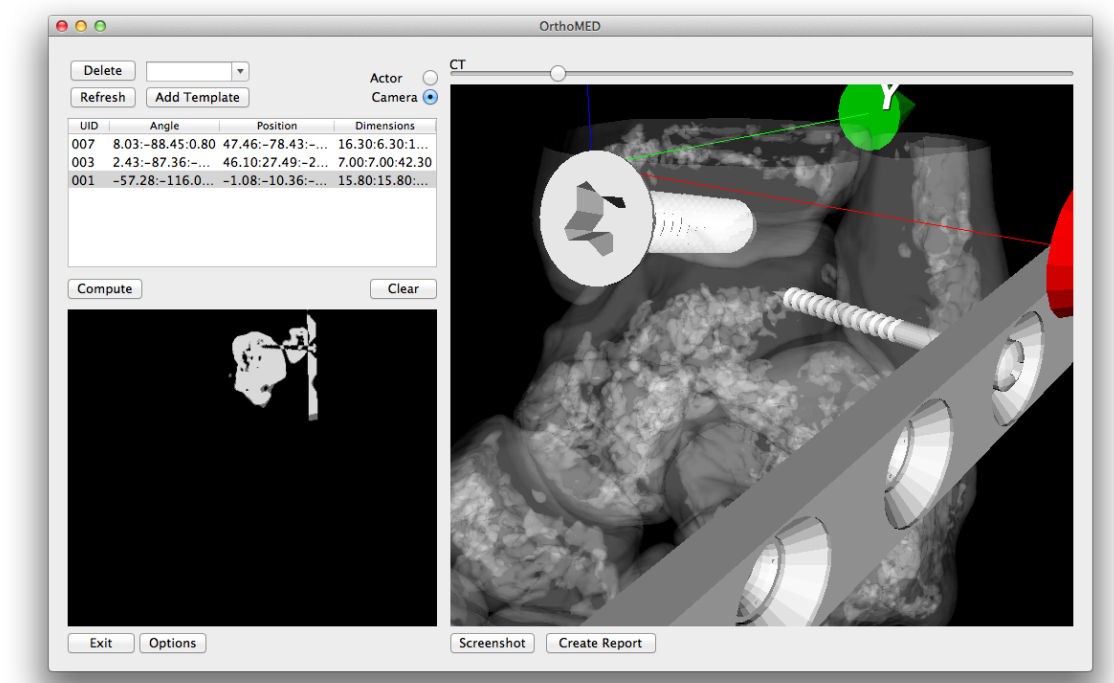

Fig. 7 OrthoMED's main window with the 3D view zoomed. 
The two radio buttons on the side are used to select which structure the user wants to move. If 'Actor' is selected, then the surgeon can select, move or rotate any independent 3D object (i.e., any template added). If 'Camera' is selected, then the point of view from all scene is moved or rotated. Figure 8 presents the window with all available templates. Here the user can select a template and check its data on the bottom. Double-clicking the chosen template, it will automatically be added to the main section, in the $(0,0,0)$ position.

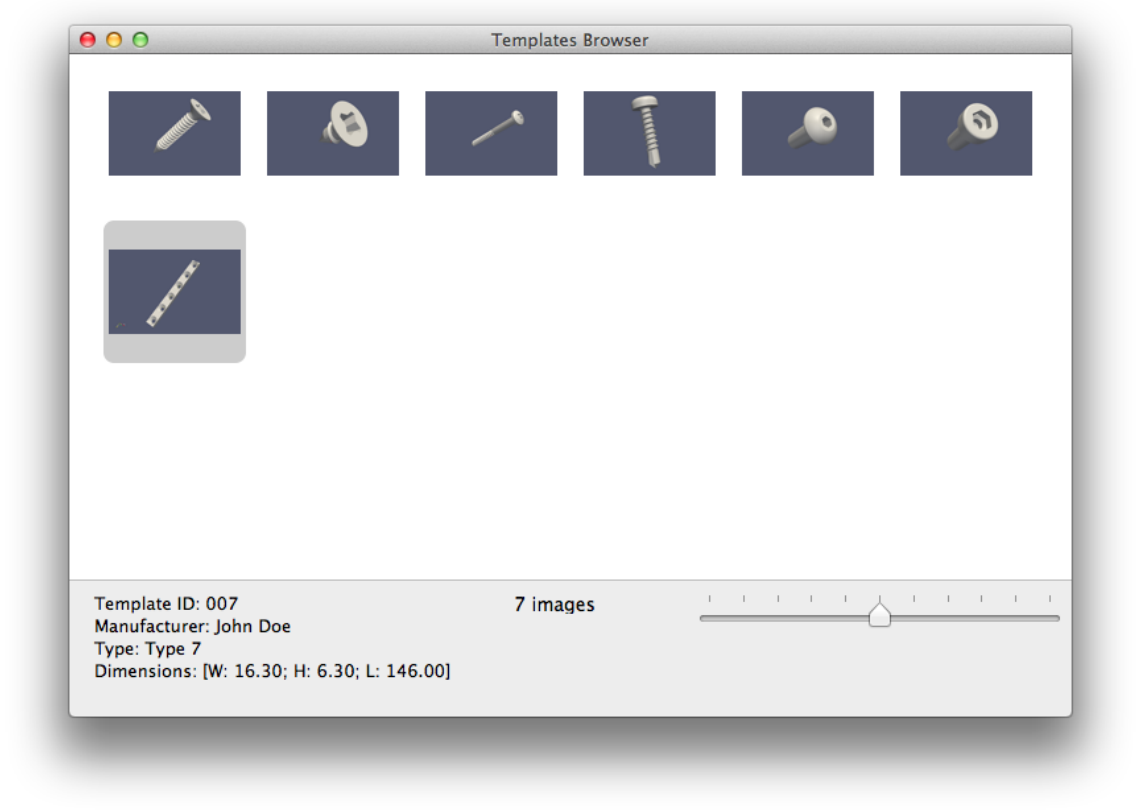

Fig. 8 OrthoMED's templates database window.

In the main section in Figure 6, one can see an isosurface of value 300 in $\mathrm{HU}$ scale. Then, some templates were added and positioned as wanted (Figure 9). On the left side we have a table with some spacial information about the templates, e.g., their position in the 3D space and angle with the isosurface. Below that table there is a section with that same 3D model including templates but now sliced in an axial plane for a better analysis of the templates real positioning. This plane can be selected on the options window (Figure 10). 


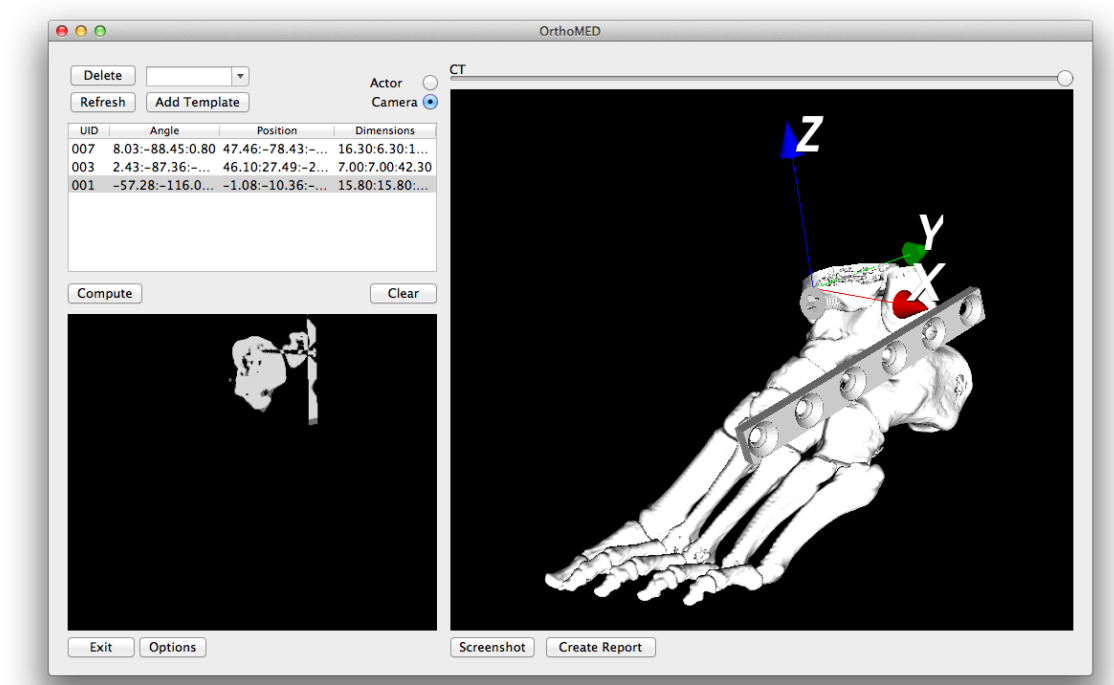

Fig. 9 OrthoMED's main window with some templates on the generated isosurface.

\begin{tabular}{|l|l|}
\hline$\odot 00$ & Options \\
\hline Isosurfaces: \\
$\checkmark$ Bone $\square$ Skin $\square$ Vessel \\
$\square$ Choose: \\
\hline Slice Mode: \\
Axial $\bigcirc$ Coronal $\bigcirc$ Sagittal \\
Oblique \\
\hline
\end{tabular}

Fig. 10 Options window.

Figure 11 presents some elements of the array with the slices of the final 3D model. Zooming and decreasing the opacity of the isosurface, the surgeon is able to analyze the exact spot where the template is positioned (Figure 7). Figure 12 shows a new isosurface added. In that way, the surgeon is now able to evaluate about his plan and how it will affect the surrounding tissues. This is quite useful for his analysis. This isosurface and its value could be entered in the options window (Figure 10). 


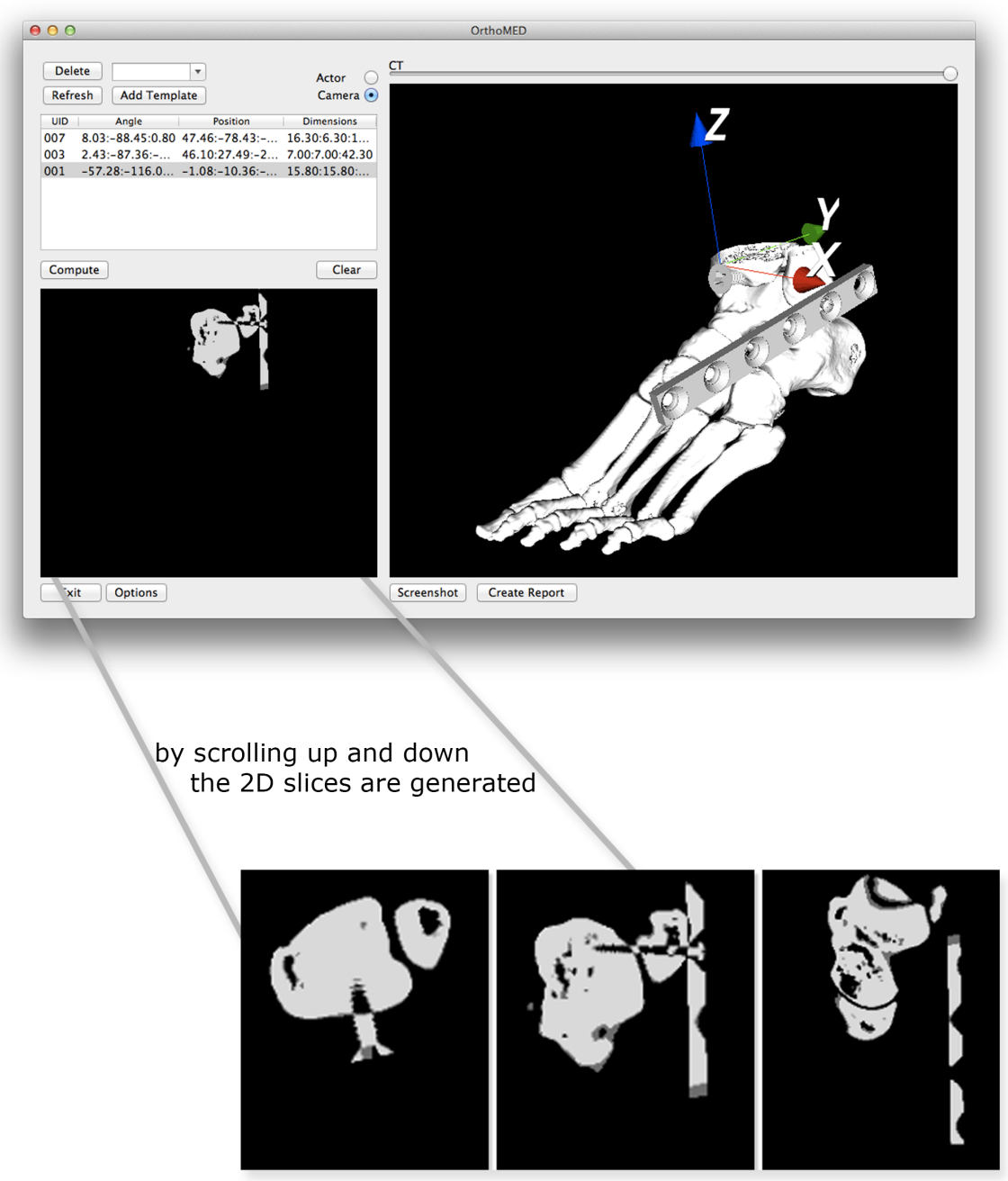

Fig. 11 2D slices generated from the planned 3D model. 


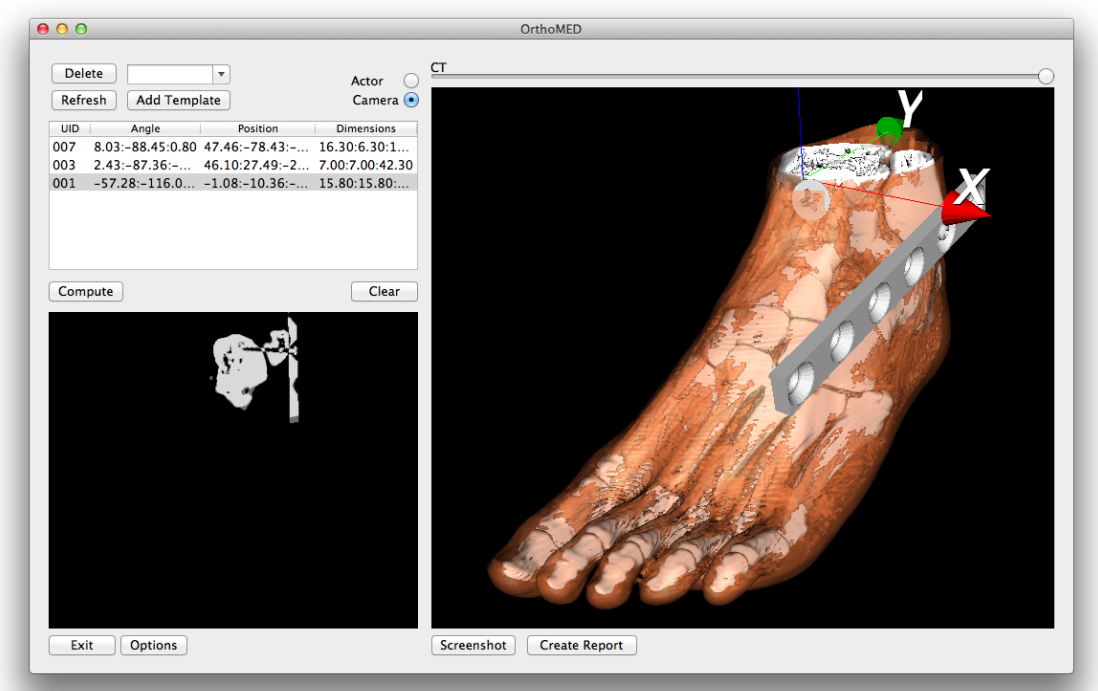

Fig. 12 OrthoMED's main window with the generated isosurface.

After the planning process, the surgeon is able to export a report with all the information needed, e.g., a table with the templates information and their position in the 3D space, some screenshots taken from the final 3D model as well as the patient's specific information (Figure 13). 


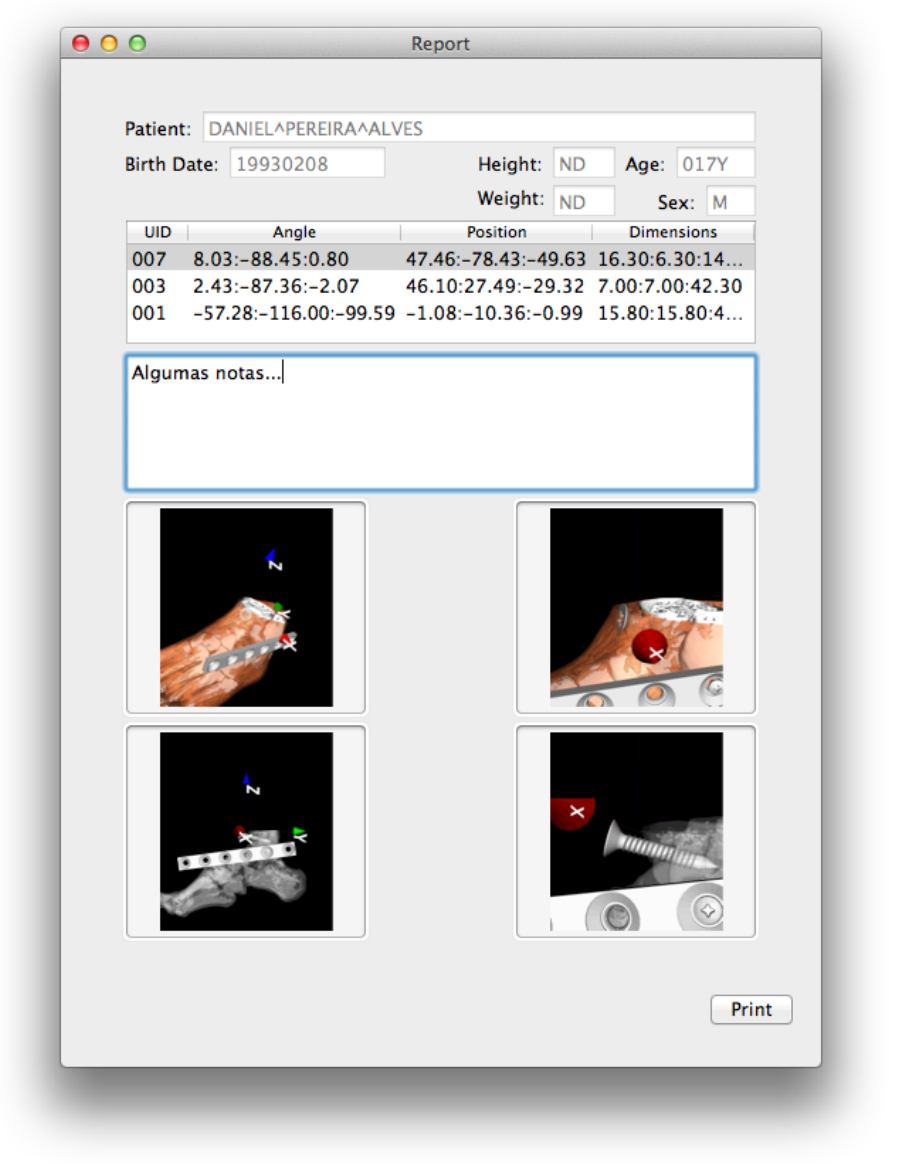

Fig. 13 Surgery planning report export window.

\section{Conclusion/Discussion}

The main goal of the presented work is to create a solution which allows the interoperability between images in two different format types. The currently available solutions have limitations, some of them do not include 3D modeling or when is that available, the creation of the model is based on standard models or the intervention of specialized technicians is required. The OrthOMED plugin enables the interoperability between a CT scan 3D model and surgical templates representing orthopedic physical implants. Since we are dealing with different types of image (i.e. the CT study is a matrix of voxels and the surgical templates are vector graphics) it was necessary to develop a method to join them in the same planes. These surgical templates 
are STL files, created from CAD models of physical implants provided by medical implants suppliers. The advantage in using this kind of files is due to its wide use by the implants industry. Comparing the proposed solution with the described commercial ones, OrthoMED presents an alternative approach, delegating the task entirely to the surgeon. With this plugin, the surgeon is able to add templates and handle their position always in a 3D space, which allows a constant evaluation of the best positioning. He can also slice the final 3D model with templates on it. After choosing the slicing plane (e.g. Axial, Sagittal, Coronal, Oblique), the surgeon can evaluate his surgical solution in a $2 \mathrm{D}$ view that gives him more detailed information. Finally he can export the surgery report plan. The developed solution becomes advantageous for the surgeons. Thus, they can manipulate the generated 3D model, composed by one or more isosurfaces, and they can add the implants templates from the database. Comparing this solution with others, OrthoMED brings a different, enhanced and complementary solution to orthopedic surgical planning.

\section{Future Work}

The OrthoMED plugin can already be considered an advantageous tool for the surgeons. However, this application can be improved whether some features were added. It will be relevant to provide some image processing algorithms such as image segmentation. When there are fragments of free bone tissue in the muscle of the patient due to an injury, it is necessary for the surgeon to select and manipulate, thus being able to use the implants for reconstruction of the affected area. Other important feature is to highlight the intersection area, determining the points where the intersections occur. Furthermore, implementing more 3D modeling algorithms, the surgeons has the possibility to decide and choose the most suitable tool for the planning of each case. Finally, it is important to improve the user interface and user experience of the application, making it more intuitive and simpler to operate.

\section{References}

[1] Bianchi A, Muyldermans L, Martino MD, Lancellotti L, Amadori S, Sarti A, Marchetti C (2010) Facial Soft Tissue Esthetic Predictions: Validation in Craniomaxillofacial Surgery With Cone Beam Computed Tomography Data. Journal of Oral and Maxillofacial Surgery 68(7):1471-1479, DOI http://dx.doi.org/10.1016/j.joms.2009.08.006

[2] Fuchs H, Kedem ZM, Uselton SP (1977) Optimal surface reconstruction from planar contours. ACM SIGGRAPH Computer Graphics 11(2):236-236, DOI 10.1145/965141.563899

[3] Hak DJ, Rose J, Stahel PF (2010) Preoperative planning in orthopedic trauma: benefits and contemporary uses. Orthopedics 33(8):581-4, DOI 
10.3928/01477447-20100625-21

[4] Hsu AR, Kim JD, Bhatia S, Levine BR (2012) Effect of training level on accuracy of digital templating in primary total hip and knee arthroplasty. Orthopedics 35(2):e179-83, DOI 10.3928/01477447-20120123-15

[5] Hu Y, Li H, Qiao G, Liu H, Ji A, Ye F (2011) Computer-assisted virtual surgical procedure for acetabular fractures based on real CT data. Injury 42(10):1121-4, DOI 10.1016/j.injury.2011.01.014

[6] Kitware I (2014) Itk - insight segmentation and registration toolkit. URL http://www.itk.org, accessed: 2014-03-31

[7] Kitware I (2014) Vtk - visualization toolkit. URL http://www.vtk.org, accessed: 2014-03-31

[8] Lattanzi R, Viceconti M, Petrone M, Quadrani P, Zannoni C (2002) Applications of 3D medical imaging in orthopaedic surgery:introducing the hip-op system. In: Proceedings. First International Symposium on 3D Data Processing Visualization and Transmission, IEEE Comput. Soc, pp 808-811, DOI 10.1109/TDPVT.2002.1024165

[9] Lorensen WE, Cline HE (1987) Marching cubes: A high resolution 3D surface construction algorithm. ACM SIGGRAPH Computer Graphics 21(4):163-169

[10] Mabrey JD, Reinig KD, Cannon WD (2010) Virtual reality in orthopaedics: is it a reality? Clinical orthopaedics and related research 468(10):2586-91, DOI 10.1007/s11999-010-1426-1

[11] Malaterre M, et al JPR (2014) Gdcm - grassroots dicom library. URL http://gdcm.sourceforge.net/, accessed: 2014-03-31

[12] Rosset A (2014) Osirix imaging software - advanced open-source pacs workstation dicom viewer. URL http://www.osirix-viewer.com, accessed: 2014-0331

[13] Rosset A, Spadola L, Ratib O (2004) OsiriX: an open-source software for navigating in multidimensional DICOM images. Journal of digital imaging : the official journal of the Society for Computer Applications in Radiology 17(3):205-16, DOI 10.1007/s10278-004-1014-6

[14] Roth SD (1982) Ray casting for modeling solids. Computer Graphics and Image Processing 18(2):109-144, DOI http://dx.doi.org/10.1016/0146664X(82)90169-1

[15] Shiha A, Krettek C, Hankemeier S, Liodakis E, Kenawey M (2010) The use of a professional graphics editing program for the preoperative planning in deformity correction surgery: A technical note. Injury 41(6):660-4, DOI 10.1016/j.injury.2009.10.051

[16] Sikorski JM, Chauhan S (2003) Aspects of current management. The Journal of bone and joint surgery 85(3):319-323

[17] Steen A, Widegren M (2013) 3D Visualization of Pre-operative Planning for Orthopedic Surgery. In: T Ropinski and J Unger (ed) Proceedings of SIGRAD 2013, Visual Computing, June 13-14, Linköping University Electronic Press, Linköpings universitet, Norrköping, Sweden, pp 1-8 
[18] Steinberg EL, Shasha N, Menahem A, Dekel S (2010) Preoperative planning of total hip replacement using the TraumaCad system. Archives of orthopaedic and trauma surgery 130(12):1429-32, DOI 10.1007/s00402-010-1046-y

[19] Suero EM, Hüfner T, Stübig T, Krettek C, Citak M (2010) Use of a virtual 3D software for planning of tibial plateau fracture reconstruction. Injury 41(6):589-91, DOI 10.1016/j.injury.2009.10.053

[20] The B, Verdonschot N, van Horn JR, van Ooijen PMA, Diercks RL (2007) Digital Versus Analogue Preoperative Planning of Total Hip Arthroplasties: A Randomized Clinical Trial of 210 Total Hip Arthroplasties. The Journal of Arthroplasty 22(6):866-870, DOI http://dx.doi.org/10.1016/j.arth.2006.07.013

[21] Wade RH, Kevu J, Doyle J (1998) Pre-operative planning in orthopaedics: a study of surgeons' opinions. Injury 29(10):785-6

[22] Wang H (2009) Three-Dimensional Medical CT Image Reconstruction. In: 2009 International Conference on Measuring Technology and Mechatronics Automation, Ieee, pp 548-551, DOI 10.1109/ICMTMA.2009.10 